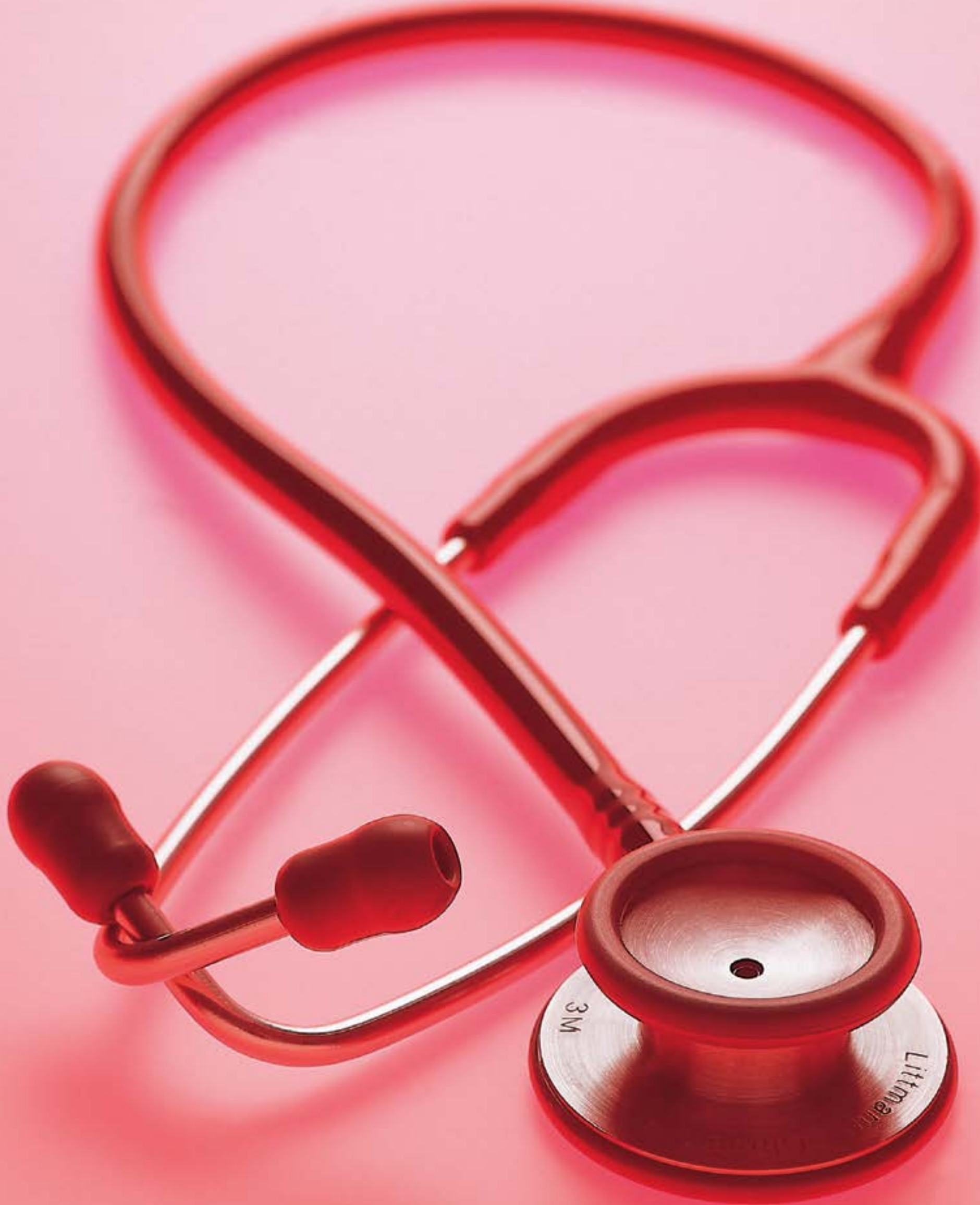




\title{
Auf den Ton kommt es an Auskultation von Herz und Lunge
}

\author{
Giemen, Pfeifen, Rumpeln, Rasseln - die Bandbreite möglicher Auskulta- \\ tionsbefunde ist groß und mag auf den ersten Blick unüberschaubar \\ wirken. Trotzdem sollte man sich die Mühe machen, das richtige Zuordnen \\ der Geräusche und Töne zu üben: Die Auskultation liefert ein wichtiges \\ Puzzleteil für die Diagnostik.
}

Fallbeispiel Sie werden von ihrer Leitstelle zu einem Patienten mit „Atembeschwerden“ alarmiert. Nach einer kurzen Anfahrt erreichen Sie einen Wohnblock und machen sich auf den Weg in den 3. Stock. Die Wohnungstür steht bereits offen, und Sie folgen einer Sauerstoffleitung quer durch die Wohnung in das Wohnzimmer. Auf der Couch finden Sie einen etwa 65-jährigen Patienten mit Tachypnoe und keuchender Ausatmung vor. Eine Zyanose sehen Sie nicht. Der Patient berichtet Ihnen in abgehackten Sätzen, dass die Luftnot schon seit mehreren Tagen zunehmen würde und er es nun nicht mehr ausgehalten habe. Er könne seit ein paar Tagen auch sehr schlecht schlafen, da die Luftnot im Liegen zunähme und von einem trockenen, unproduktiven Husten begleitet sei. Schmerzen habe er nicht. Seine Haut ist trocken und nicht überwärmt. Der Puls ist regelmäßig, tachykard und schwach tastbar.

Erstes Vorgehen Schon in diesem Moment macht der Patient auf Sie einen kranken, aber nicht kritischen Eindruck.

- Aufgrund seiner abgehackten Sprechweise und der schon ohne Stethoskop wahrnehmbaren Atemnebengeräusche vermuten Sie ein eventuelles Problem der Atemwege (A) und auch der Atmung (B).

- Sie bitten ihren Kollegen, dem Patienten eine Sauerstoffmaske mit einem Flow von 101/min zu verabreichen und somit die Dyspnoe zu lindern.

- Währenddessen möchten Sie Ihr ABCDE-Schema abschließen und beginnen mit der Auskultation der Lunge.

\section{Pulmonale Auskultation}

\section{Einführung in die Physik der Geräusche}

Strömungsgeräusche Strömungsgeräusche entstehen immer dann, wenn aus einer laminaren Strömung eine turbulente wird. Dies geschieht, wenn sich die Strombahn verengt (z.B. durch Schleim) oder aufgabelt (z.B. bei den Bronchialverästelungen).

Atemgeräusche Bei der Auskultation der Lunge mit dem Stethoskop sind üblicherweise zunächst normale Atemgeräusche zu hören, die sich je nach Ort der Auskultation etwas unterscheiden.

- In den zentralen Atemwegen (also am Hals sowie über und neben dem Brustbein) ist dies das zentrale Atemgeräusch, auch Bronchialatmen genannt. Es ist laut, klingt scharf oder rau und ist beim Ein- und Ausatmen hörbar.

- In der Peripherie sind die Geräusche der kleinen Bronchioli hörbar. Sie klingen tiefer und weniger laut als das Atemgeräusch über den zentralen Atemwegen, da sie durch das Lungengewebe abgedämpft werden. Am besten zu hören sind sie in den Phasen relativ schnellen Luftstroms, also während der Einatmung und zu Beginn der Ausatmung. Dieses „normale“ Geräusch der Luftströmung wird als vesikuläres Atemgeräusch bezeichnet.

Veränderungen der Bronchialgeräusche weisen auf Bronchialerkrankungen hin. So führt die Verengung beim Asthmaanfall zu einem verlängerten Exspirium oder aber zu einer „silent lung“, die ein fälschlich beruhigendes Fehlen typischer Befunde zur Folge hat („silent lung“: massive Überblähung mit sehr kleinen Hubvolumina und starker Abschwächung des Geräusches durch die größere Distanz der Stethoskopmembran zu den Bronchioli). 
Dämpfung Ein lokal umgrenztes Fehlen oder eine Abschwächung der Atemgeräusche tritt bei lokaler Dämpfung auf, z. B. durch einen Pleuraerguss oder einen Pneumothorax. Sind zentrale Atemgeräusche in der Peripherie zu hören, so liegt dies daran, dass z. B. eine Pneumonie oder Atelektase die Dämpfung aufhebt.

Atemgeräusche klingen auch über der gesunden Lunge nicht überall identisch. Es ist wichtig, zu wissen, welche anatomischen Strukturen sich unter der Oberfläche verbergen und ob das Atemgeräusch, dass man wahrnimmt, mit dem erwarteten übereinstimmt.

Zusätzliche Geräusche Zusätzliche Geräusche werden unterteilt nach ihrer Art und Weise. Feucht klingende, nicht kontinuierliche Geräusche unterschiedlicher Grobheit weisen auf Sekret oder Schleim hin. Je feinblasiger sie klingen, desto peripherer sind sie zu vermuten. Durch kräftiges Abhusten des Schleims lassen sich diese Rasselgeräusche häufig beenden.

Kontinuierliche Geräusche Kontinuierliche Geräusche entstehen nicht nur in, sondern auch an den Bronchien. Neben meist zähflüssigem Schleim sind Bronchialwandschwellungen oder -spasmen die Ursache.
- Brummen ist niederfrequent, laut und weist auf die geräuscherzeugenden, schwingenden Schleimfäden der großen Atemwege hin.

- Pfeifen ist ein hochfrequentes Geräusch, das durch ausgeprägte Verengungen der Strömungswege entsteht, wie beim Asthma oder der COPD (chronisch obstruktive Lungenerkrankung).

- Giemen ist ein etwas satteres Pfeifen.

Häufig sind diese Geräusche nur in der Ausatmung zu hören, da die bei der Inspiration erweiterten Atemwege die klangbildende Verengung aufheben, das Zusammenfallen des Gewebes beim Ausatmen jedoch den Durchmesser der Luftwege wieder verkleinert. Die kontinuierlichen Geräusche werden auch als Stridor bezeichnet und weisen je nach Atemphase ihres Auftretens und Ort der maximalen Projektion u.U. auf ganz unterschiedliche Verlegungen der Strombahn hin: So kann ein inspiratorischer Stridor der Nase durch eine Scheidewandverkrümmung entstehen, einer des Rachens durch Fremdkörperaspiration oder eine subglottische Stenose wie beim Pseudokrupp-Anfall. Wieder entsteht das lauteste Geräusch bei der schnellsten Strömung am Hindernis:

- Sind die oberen Atemwege verlegt, entsteht das Atemgeräusch beim (verzweifelten) Luftholen.

- Bei Verlegung der unteren Atemwege verursacht das Hinauspressen der Luft das Geräusch.

\section{Tabelle 1 Auswahl relevanter Diagnosen und deren pathologische Auskultationsbefunde}

\begin{tabular}{|c|c|c|c|c|}
\hline Diagnose & patholog. Atemgeräusch & Lokalisation & assoziierte Symptome/Anamnese & weiterführende Diagnostik \\
\hline Pneumonie & feuchte Rasselgeräusche & $\begin{array}{l}\text { meist lokal begrenzt } \\
\text { und seitenbetont }\end{array}$ & $\begin{array}{l}\text { schleichender Beginn, Bettlägerigkeit, kürzlich } \\
\text { stationärer Aufenthalt, persistierender Infekt, } \\
\text { produktiver Husten, Fieber, Schwäche }\end{array}$ & $\begin{array}{l}\text { Temperatur, Atemfrequenz, Herzfrequenz, } \\
\text { Blutdruck, } \mathrm{SpO}_{2} \text { (periphere Sauerstoffsättigung) }\end{array}$ \\
\hline Lungenödem & feuchte Rasselgeräusche & $\begin{array}{l}\text { bilaterales Auftreten } \\
\text { (zu Beginn oft nur } \\
\text { basal) }\end{array}$ & $\begin{array}{l}\text { Belastungsdyspnoe, Orthopnoe, Angina } \\
\text { Pectoris, Zeichen der Herzinsuffizienz, Dauer- } \\
\text { medikation }\end{array}$ & $\begin{array}{l}\text { Blutdruck, EKG, Atemfrequenz, Herzfrequenz, } \\
\text { körperliche Untersuchung, } \mathrm{SpO}_{2}\end{array}$ \\
\hline Asthma bronchiale & Giemen, Brummen & $\begin{array}{l}\text { ubiquitär, also nicht } \\
\text { örtlich begrenzt }\end{array}$ & $\begin{array}{l}\text { plötzlicher Beginn oder anfallsartiger Charakter, } \\
\text { evtl. Auslöser bekannt, Dauermedikation, } \\
\text { Körperhaltung, verlängertes Exspirium }\end{array}$ & $\begin{array}{l}\text { Herzfrequenz, Atemfrequenz, EKG, Blutdruck, } \\
\text { Kapnografie, } \mathrm{SpO}_{2}\end{array}$ \\
\hline$C O P D$ & $\begin{array}{l}\text { Brummen, Giemen, evtl. } \\
\text { feuchte Rasselgeräusche }\end{array}$ & ubiquitär & $\begin{array}{l}\text { häufig schleichender Beginn, Dauermedikation, } \\
\text { evtl. Heimsauerstoff, Infektzeichen }\end{array}$ & Herzfrequenz, Temperatur, $\mathrm{SpO}_{2}$ \\
\hline Lungenemphysem & $\begin{array}{l}\text { häufig abgeschwächtes } \\
\text { Atemgeräusch }\end{array}$ & ubiquitär & $\begin{array}{l}\text { Anamnese, evtl. auch COPD bekannt, evtl. } \\
\text { Heimsauerstoff }\end{array}$ & s. $C O P D$ \\
\hline Bronchitis & Giemen, Brummen & meist lokal begrenzt & $\begin{array}{l}\text { Atemwegsinfekt, anfangs trockener Husten (be- } \\
\text { sonders auch nächtliche Hustenattacken) später } \\
\text { auch produktiver Husten möglich, Fieber }\end{array}$ & Temperatur, Herzfrequenz, Atemfrequenz, $\mathrm{SpO}_{2}$ \\
\hline Pneumothorax & $\begin{array}{l}\text { abgeschwächtes bzw. auf- } \\
\text { gehobenes Atemgeräusch } \\
\text { (unilateral) }\end{array}$ & $\begin{array}{l}\text { unilateral (selten } \\
\text { bilateral } \rightarrow \text { schweres } \\
\text { Thoraxtrauma) }\end{array}$ & $\begin{array}{l}\text { Verletzungsmechanismus, bekannte COPD, } \\
\text { häufig auch junge, schlanke Patienten betroffen, } \\
\text { Raucher }\end{array}$ & $\begin{array}{l}\text { Atemfrequenz, Herzfrequenz, Blutdruck, } \\
\text { Perkussion, Thorax, } \mathrm{SpO}_{2} \\
\text { Cave: Bei fehlenden peripheren Pulsen, Bewusst- } \\
\text { seinsstörung und/oder gestauten Halsvenen } \\
\text { erwäge Spannungspneumothorax. }\end{array}$ \\
\hline Lungenembolie & $\begin{array}{l}\text { normale Atmung, evtl. } \\
\text { beginnendes Giemen }\end{array}$ & - & $\begin{array}{l}\text { Hämoptysis, Thoraxschmerz (häufig atem- } \\
\text { abhängig), neu aufgetretene Rhythmusstörung, } \\
\text { Zeichen der akuten Rechtsherzinsuffizienz }\end{array}$ & $\begin{array}{l}\text { Atemfrequenz, Blutdruck, EKG, Risikofaktoren, } \\
\mathrm{SpO}_{2}\end{array}$ \\
\hline $\begin{array}{l}\text { Anaphylaxie } \\
\text { (Stridor als Leit- } \\
\text { symptom) }\end{array}$ & $\begin{array}{l}\text { Giemen, verlängertes } \\
\text { Exspirium, evtl. auch } \\
\text { inspiratorischer Stridor }\end{array}$ & ubiquitär & $\begin{array}{l}\text { akuter Beginn und rasche Progression, Antigen- } \\
\text { kontakt (bekannt oder unklar), Quinckeödem, } \\
\text { Flush, Urtikaria, Juckreiz, vegetative Symptome, } \\
\text { Schock }\end{array}$ & Atemfrequenz, Blutdruck, Herzfrequenz, $\mathrm{SpO}_{2}$ \\
\hline
\end{tabular}


Ein inspiratorischer Stridor weist also auf eine Verlegung der oberen Atemwege hin - mit Lokalisation meist zwischen Kopf und oberer Thoraxöffnung. Wichtige Diagnosen und den dazugehörigen pathologischen Auskultationsbefund zeigt $\bullet$ Tab. 1.

Dokumentation Dokumentieren Sie alle Befunde, auch wenn sie zunächst unauffällig sind. Nur dadurch lässt sich der Verlauf beobachten. Eine beschreibende Formulierung ist wichtig, keine bewertende. Es macht wenig Sinn, im Protokoll zu vermerken, die Lunge klinge „gut“. Vielmehr sollen der Grundklang und eventuelle Nebengeräusche notiert werden, z.B. in gebräuchlichen Abkürzungen: „VAG bds., keine NG“ drückt platzsparend aus, dass die Lunge auf beiden Seiten ein vesikuläres Atemgeräusch ohne auffällige Nebengeräusche zeigt.

Die manchmal verwendete Formulierung „opB“ (ohne pathologischen Befund) ist angesichts der oft unruhigen Umgebungsbedingungen und der Hektik am Einsatzort sehr unsicher und nicht empfehlenswert.

\section{Infobox: kardiales Lungenödem}

Beim kardialen Lungenödem kommt es durch eine verminderte Pumpleistung des linken Herzens zu einem Rückstau von Blut in den Lungenkreislauf. Der daraus resultierende Druck im Kapillargebiet der Lungengefäße führt zu einem Übertritt von Flüssigkeit aus dem Intravasalraum in das Interstitium und letztlich in die Alveolen. Dieser Ablauf der Pathophysiologie führt zu verschiedenen Atemnebengeräuschen in den einzelnen Phasen:

- interstitielle Phase: Durch die Füssigkeitsansammlungen im Interstitium werden kleine Atemwege eingeengt, es kann zum sog. „Asthma cardiale“ mit giemenden Atemgeräuschen kommen.

- alveoläres Lungenödem: Durch den Übertritt der Flüssigkeit in die Alveolen kommt es zu den klassischen feuchten Rasselgeräuschen. Diese sind jedoch in der Entstehungsphase häufig nicht ohne Stethoskop zu hören. Typischerweise beginnen die Rasselgeräusche beidseits in den basalen Lungenabschnitten, da hier durch die Schwerkraft der Druck in den Gefäßen am höchsten ist.

Cave Die Entstehung der verschiedenen Geräusche zeigt, dass ein bestimmter Befund nicht automatisch eine dazugehörige Diagnose liefert. Die Auskultation kann helfen, eine Verdachtsdiagnose zu bestätigen oder auszuschließen. Sie ist also ein Puzzleteil in der Diagnostik, ist aber ohne adäquate Anamnese nur bedingt aussagekräftig.
Um physiologische oder pathologische Atemgeräusche überhaupt sicher diagnostizieren zu können, ist ein strukturiertes Vorgehen erforderlich. Dieses wird im Beitrag „Schritt für Schritt: pulmonale und kardiale Auskultation“ o ab S. 144 vorgestellt.

Fortsetzung Fallbeispiel Bei der Auskultation stellen Sie eine verlängerte exspiratorische Phase sowie ein Giemen fest. Aufgrund der bisherigen Anamnese drängt sich Ihnen der Verdacht auf, dass es sich um eine infektexazerbierte COPD (Chronic Obstructive Pulmonary Disease: Chronisch obstruktive Lungenerkrankung) handeln könnte. Als ihr Kollege Ihnen jedoch den Medikamentenplan des Patienten reicht, werden Sie stutzig: Als Dauermedikation sind ein ACE-Hemmer (ACE: Angiotensin Converting Enzyme), ein Kalzium-Antagonist und ein Diuretikum verschrieben - keines der erwarteten bronchodilatorisch wirksamen Medikamente, die für eine COPD typisch wären. Trotz des Ihnen bekannten Auskultationsbefunds stellen Sie die Diagnose erst einmal zurück, um sich weitere Informationen zu beschaffen und entschließen sich dazu, die Herztöne des Patienten zu auskultieren.

\section{Herztöne, Herzgeräusche}

\section{Physik der Geräusche}

Ton oder Geräusch? Man unterscheidet bei der Auskultation des Herzens zwischen Herztönen und Herzgeräuschen - wenngleich physikalisch betrachtet beide Schallphänomene Geräusche sind. Die etwas reiner klingenden, physiologischen Herztöne lassen sich gut den mechanischen Aktionen des Herzens zuordnen. Der 1. Ton entspricht der Vibration durch die Stauchung der Blutsäule bei der Kontraktion des Myokards in der Anspannungsphase der Systole. Der 2. Herzton entspricht dem Schluss der Taschenklappen am Ende der Austreibungsphase.

- Töne sind also keine Flussgeräusche.

- Geräusche entstehen erst durch turbulenten Fluss - z.B. durch Verengung einer Herzklappe oder durch ein erhöhtes Herzminutenvolumen.

Systolische und diastolische Geräusche Herzgeräusche zwischen dem 1 . und 2 . Ton bezeichnet man als systolische Geräusche und solche zwischen dem 2 . und 1 . Ton als diastolische. Um ein Geräusch sicher einer Klappe zuordnen zu können, ist zum einen die Suche nach dem Punkt der besten Hörbarkeit (punctum 
Tabelle 2 Strömungsgeräusche und allgemeines, klinisches Erscheinungsbild

\begin{tabular}{llll} 
Klappe & Geräusch & Projektion & Allgemeine Symptome \\
\hline diastolische Geräusche & & \\
\hline Aorteninsuffizienz & hauchend, gießend, abnehmend durch & 3. ICR links parasternal (Erb-Punkt) & große Blutdruckamplitude, blasse Haut, Herzinsuffizienz \\
\hline Mitralstenose & rumpelnd, paukender 1. Herzton & 5. ICR, besonders in Linksseitenlage & $\begin{array}{l}\text { absolute Arrhythmie, Dyspnoe, Asthma cardiale, } \\
\text { Leistungsminderung, periphere Zyanose }\end{array}$ \\
\hline systolische Geräusche & & 2. ICR rechts parasternal Carotiden & Belastungsdyspnoe, Angina Pectoris, Synkopen bei Belastung \\
\hline Aortenstenose & rau, spindelförmig, schwirrend & 5. ICR links parasternal linke Axilla & $\begin{array}{l}\text { Dyspnoe, Herzklopfen, Asthma cardiale (mit nächtlichem Hus- } \\
\text { ten), Lungenödem und kardialer Schock bei Dekompensation }\end{array}$ \\
\hline Mitralinsuffizienz & hochfrequent, ganze Systole hindurch & &
\end{tabular}

maximum, p.m.) nötig, zum anderen die Palpation des Pulses während der Auskultation. Gerade bei höheren Herzfrequenzen lässt sich nur so eine sichere Einteilung in systolische und diastolische Herzgeräusche treffen.

Strömungsgeräusche durch Herzklappenfehler Bei einer Insuffizienz schließt die Klappe nicht korrekt und dichtet somit nicht vollständig ab. Bei einer Stenose öffnet sie nicht vollständig, und der Auswurf wird behindert. In beiden Fällen entstehen Turbulenzen, die bei der Auskultation als Strömungsgeräusche hörbar sind. Die notfallmedizinisch relevanten Klappendefekte der symptomatischen Herzfehler sind nach Häufigkeit:

- Aortenklappenstenose (45\%)

- Mitralinsuffizienz (35\%)

- Aortenklappeninsuffizienz (10\%)

- Mitralstenose (10\%)

Diese Klappenfehler sind in Europa meist degenerativ, also Erkrankungen des Alters. Klappenfehler des rechten Herzens sind sehr selten und treten durch Entzündungen auf (z.B. bei i.v. Drogenkonsum) oder als beteiligte Klappen bei anderen Herzfehlern (Vitien).

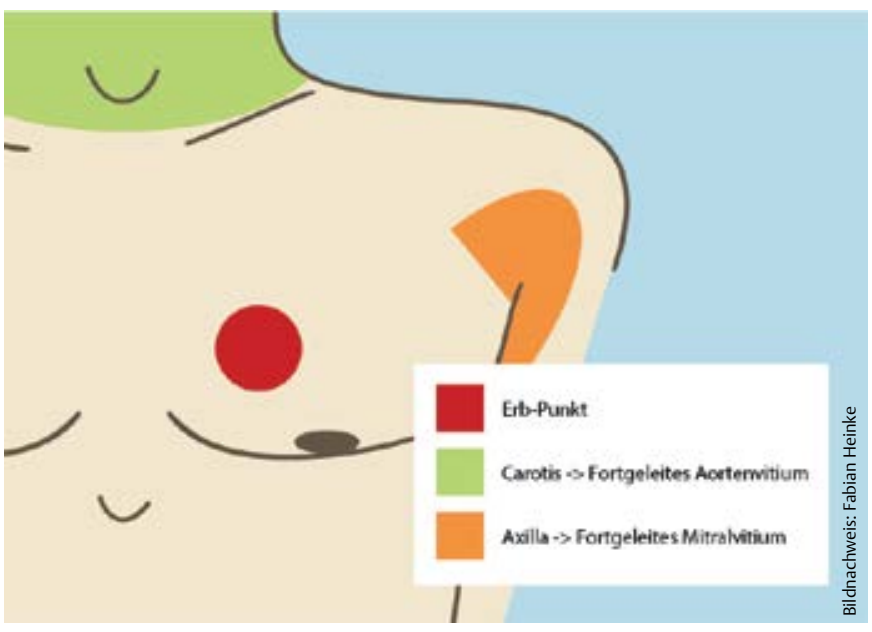

Abb. 1 Herztöne lassen sich im Überblick gut über dem Erb-Punkt auskultieren. Fortgeleitete Strömungsgeräusche geben Auskunft darüber, ob das Strömungsgeräusch eher von einem Aorten- oder einem Mitralvitium stammt.
Bei der Auskultation ist es wichtig, dass Sie die Herztöne und ihre zugehörige Phase identifizieren können. Hier hilft nur: üben, üben, üben. Hören Sie routinemäßig die Herztöne Ihrer Patienten ab und üben Sie deren Zuordnung. Je sicherer Sie damit sind, umso leichter fallen Ihnen pathologische Befunde auf.

Auskultationspunkte Am einfachsten können die Herztöne über dem Erb-Punkt (3. ICR parasternal links) auskultiert werden. Dieser liegt über der Klappenebene des Herzens und alle Klappen werden nahezu gleich laut dargestellt. Deshalb lassen sich die Herztöne und eventuelle pathologische Geräusche besonders sensitiv (jedoch unspezifisch) erfassen. Um zu differenzieren, ob das Strömungsgeräusch eher von einem Aorten- oder einem Mitralvitium kommt, hilft es nach fortgeleiteten Strömungsgeräuschen zu suchen ( $\bullet$ Abb. 1 ). Der genaue Untersuchungsablauf ist im Beitrag „Schritt für Schritt: pulmonale und kardiale Auskultation ab os. 144 beschrieben.

Auskultationsbefunde Die Geräusche der Herzfehler lassen sich aus dem Verständnis der Pathophysiologie ableiten. Die Klappen sollen den Blutstrom in Abhängigkeit der Herzphase leiten. In der Systole öffnet sich daher die Aortenklappe, während die Mitralklappe geschlossen bleibt. In der Diastole ist die Mitralklappe geöffnet, Blut strömt in den Ventrikel, die Aortenklappe ist geschlossen, und die aortale Blutportion fließt in den Körperkreislauf. Da Geräusche bei turbulenter Strömung entstehen, sind es die Momente schnellen Flusses, in denen sie gut zu hören sind. Deshalb sind die systolischen Herzgeräusche Folge von Aortenstenose und Mitralinsuffizienz, die diastolischen Geräusche Folge von Aorteninsuffizienz und Mitralstenose. - Tab. 2 fasst mögliche Strömungsgeräusche zusammen. @ Abb. 2 zeigt den Zusammenhang zwischen Herzzyklus und den zugehörigen Vitien. 
Neben Geräuschphänomenen, die Vitien hervorrufen, gibt es noch einen weiteren wichtigen kardialen Auskultationsbefund: abgeschwächte Herztöne. Hierbei handelt es sich um auffällig leise Herztöne, die z.B. auf eine Herzbeuteltamponade hinweisen können. - Tab. 3 gibt einen Überblick der Diagnosen im Zusammenhang mit abgeschwächten Herztönen.

\section{$\triangle$} Ein gefundenes Vitium alleine liefert keine Diagnose, kann aber wichtige Informationen beisteuern, um Differenzialdiagnosen zu formulieren oder mögliche Komplikationen während der Versorgung frühzeitig einzukalkulieren. Deshalb ist das Entdecken eines Klappenfehlers und seine Einordnung in den Gesamtzustand des Patienten präklinisch u. U. wichtiger als die genaue Zuordnung zu einer Klappe. - Tab. 2, 3 und 4 geben wichtige Anhaltspunkte für die Differenzialdiagnostik kardialer Geräuschphänomene.

Fortsetzung Fallbeispiel Bei der Auskultation der Herztöne fällt Ihnen ein deutliches Austreibungsgeräusch in der Systole auf, das gut hörbar in die Axilla fortgeleitet wird. Sie atmen kurz durch und sind froh, nicht übereilt Ihrem ersten Impuls bei der Diagnosefindung erlegen zu sein - möglicherweise hätten $\beta$-Sympathomimetika den Patientenzustand verschlechtert.

Durch die Sauerstofftherapie geht es Ihrem Patienten schon deutlich besser. Sie melden ihn mit der Verdachtsdiagnose einer

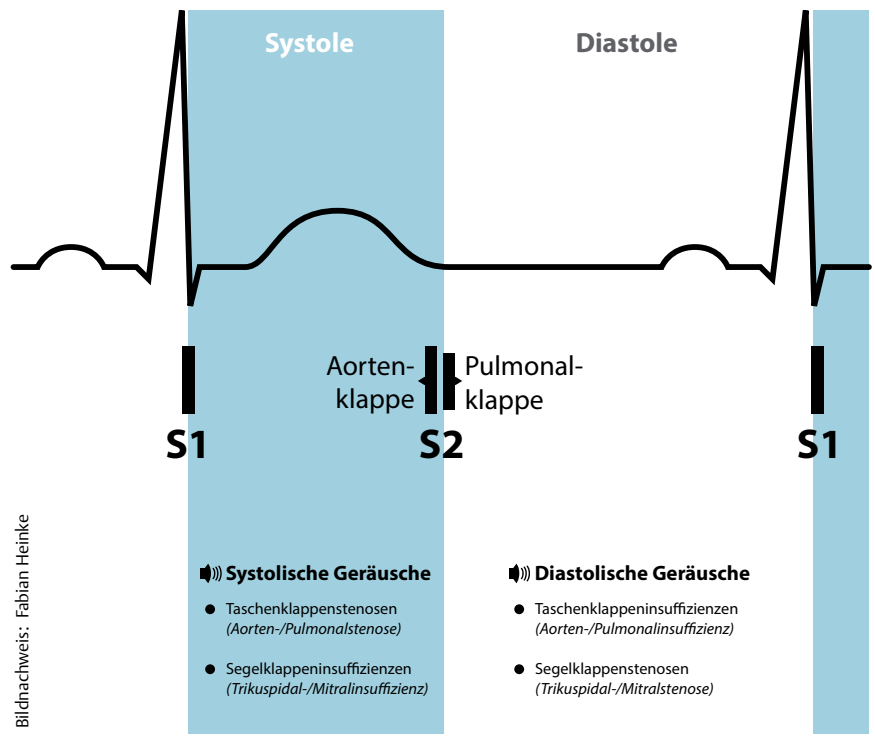

Abb. 2 Zusammenhang zwischen Herzzyklus und den zugehörigen Vitien.

\section{Tabelle 3 Notfallmedizinische Diagnosen mit abgeschwächten Herztönen}

\begin{tabular}{ll} 
Diagnose & Klinisches Erscheinungsbild \\
\hline $\begin{array}{l}\text { traumatische Peri- } \\
\text { kardtamponade }\end{array}$ & $\begin{array}{l}\text { Zeichen eines penetrierenden oder stumpfen Thoraxtraumas, Z. n. kürzlich gelegtem Herzkatheter, retrosternale Schmerzen, rasche Progression, } \\
\text { Herztöne werden im Verlauf leiser, Dyspnoe, Beck-Trias der Perikardtamponade: 1. gestaute Halsvenen; 2. Hypotension; 3. abgeschwächte Herztöne }\end{array}$ \\
\hline Perikarderguss & z.B. Patient mit Krebsleiden, V.a. Perikarditis, langsame Progression, Zeichen der Beck-Trias bei Dekompensation \\
\hline Herzinsuffizienz & bekannte höhergradige Herzinsuffizienz mit reduzierter Ejektionsfraktion (evtl. aktueller Arztbrief), Z.n. Myokardinfarkt \\
\hline
\end{tabular}

\section{Tabelle 4 Notfallmedizinische Diagnosen mit systolischen oder diastolischen Herzgeräuschen}

\begin{tabular}{|c|c|c|}
\hline Diagnose & Vitium & Klinisches Erscheinungsbild \\
\hline \multicolumn{3}{|c|}{ Notfallmedizinische Diagnosen mit systolischen Herzgeräuschen } \\
\hline Myokardinfarkt & $\begin{array}{l}\text { akute Mitralinsuffizienz bei } \\
\text { Papillarmuskelabriss }\end{array}$ & $\begin{array}{l}\text { plötzlicher Beginn, Schmerzsymptomatik eines akuten Koronarsyndroms (ACS), kardiogener Schock, } \\
\text { Dyspnoe, Lungenödem, EKG-Zeichen }\end{array}$ \\
\hline $\begin{array}{l}\text { Zustand nach rheumatischer } \\
\text { Erkrankung (z. B. Endokarditis) }\end{array}$ & Aortenstenose, Mitralinsuffizienz & $\begin{array}{l}\text { Zeichen der Linksherzinsuffizienz, Migrationshintergrund (häufig liegen zwischen der unbehandelten } \\
\text { Grunderkrankung und Erstmanifestation Jahrzehnte ohne Symptome), Abgeschlagenheit, Müdigkeit, } \\
\text { Palpitationen, beginnendes Lungenödem }\end{array}$ \\
\hline Synkope & Aortenstenose & $\begin{array}{l}\text { eher älterer Patient, Schwindel, Dyspnoe, evtl. pectanginöse Beschwerden, reduzierte Belastbarkeit, } \\
\text { Palpitationen und Arrhythmien, EKG: Zeichen der Linksherzhypertrophie }\end{array}$ \\
\hline \multicolumn{3}{|c|}{ Notfallmedizinische Diagnosen mit diastolischen Herzgeräuschen } \\
\hline Aortendissektion & akute Aorteninsuffizienz & $\begin{array}{l}\text { plötzlicher Beginn mit reißendem Schmerz im Thorax oder zwischen den Schulterblättern, hochakuter } \\
\text { Verlauf, häufig rapide/schnelle Dekompensation }\end{array}$ \\
\hline Lungenembolie & akute Pulmonalisinsuffizienz & $\begin{array}{l}\text { atemabhängiger Schmerz im Thorax, Hypotonie, Zeichen des akuten Rechtsherzversagens, EKG: } \\
\text { Rechtsherzbelastung, Tachykardie, evtl. blutig, schaumiges Sputum, plötzlicher Beginn, Risikofaktoren für } \\
\text { Tromboembolie, therapierefraktärer } \mathrm{SpO}_{2} \text {-Abfall }\end{array}$ \\
\hline $\begin{array}{l}\text { dekompensierte } \\
\text { Rechtsherzinsuffizienz }\end{array}$ & Mitralstenose & $\begin{array}{l}\text { schleichender Prozess, periphere Ödeme, Aszites, Orthopnoe, Asthma cardiale } \\
\text { Cave: Optisch und auch auskultativ kann dieser Patient schnell und fälschlich mit einem COPD-Patienten } \\
\text { verwechselt werden, hier kann der kardiale Auskultationsbefund wegweisend sein! }\end{array}$ \\
\hline
\end{tabular}




\section{Infobox: Entstehung von Klappenfehlern}

Am häufigsten entstehen Klappenfehler durch degenerative Veränderungen. Diese Vorgänge können entweder die Klappe direkt betreffen oder durch Veränderungen von Bezugsstrukturen (z. B. myokardiale Hypertrophie oder Dilatation) hervorgerufen werden. Entzündlich bedingte Klappenfehler oder angeborene Klappenfehler sind eher seltene Ursachen. Diese chronischen Herzklappenfehler sind von einer bis zu jahrzehntelangen kardialen Kompensationsphase begleitet, bevor es zur Dekompensation kommt. Im Gegensatz dazu ist der Verlauf akuter Vitien hochdynamisch und kann mit einer sofortigen Dekompensation einhergehen.

Akut auftretende Vitien sind die Insuffizienzen von Aorten- und Mitralklappe (beide kommen bei bakterieller Endokarditis vor, Insuffizienzen der Aorta auch nach Trauma oder Aortendissektion, Insuffizienzen der Mitralklappe auch nach akutem Myokardinfarkt).

dekompensierten Herzinsuffizienz in der Klinik an und können während des Transports sogar den Sauerstoffflow reduzieren. Mit einem $\mathrm{SpO}_{2}$-Wert $\left(\mathrm{SpO}_{2}=\right.$ periphere Sauerstoffsättigung) von 94\% und ohne Dyspnoe können Sie den Patienten in der Klinik übergeben.

\section{Fazit}

Gerade in der Präklinik wird die Bedeutung der Auskultation von Herz und Lunge gerne verkannt. Dabei ist sie ein unerlässlicher Baustein in der Differenzialdiagnostik vieler internistischer Notfallbilder. Sie kann Behandlungsentscheidungen unterstützen oder verändern und dient somit der richtigen Therapie - letztlich also der Patientensicherheit.

Beitrag online zu finden unter http://dx.doi.org/10.1055/s-0041-100009
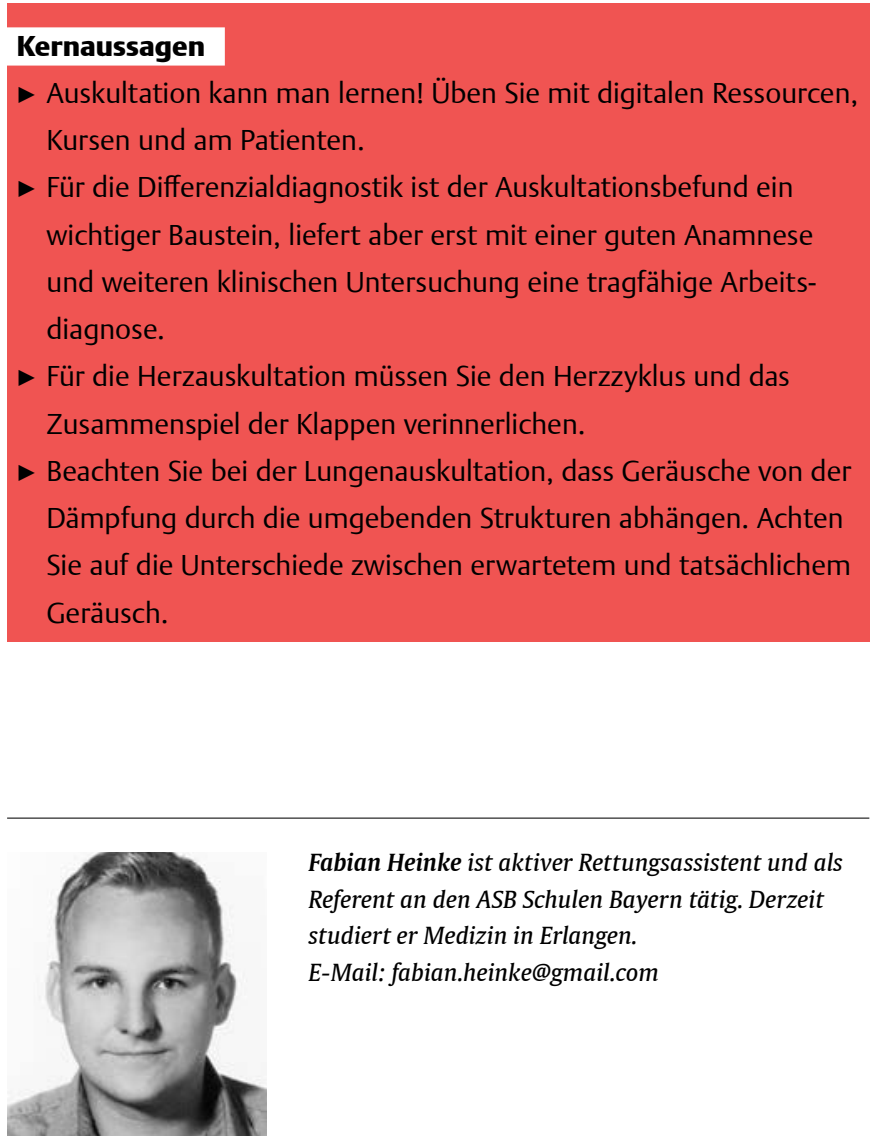

Fabian Heinke ist aktiver Rettungsassistent und als Referent an den ASB Schulen Bayern tätig. Derzeit studiert er Medizin in Erlangen. E-Mail: fabian.heinke@gmail.com

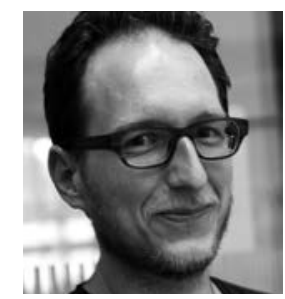

Dr. Christoph Mauer arbeitet an der Klinik für Anästhesiologie am Universitätsklinikum Erlangen. Er unterrichtet an der Rettungsdienstschule des ASB in Lauf/Pegnitz.

E-Mail: christoph.mauer@kfa.imed.uni-erlangen.de 


\section{Auf den Ton kommt es an Auskultation von Herz und Lunge}

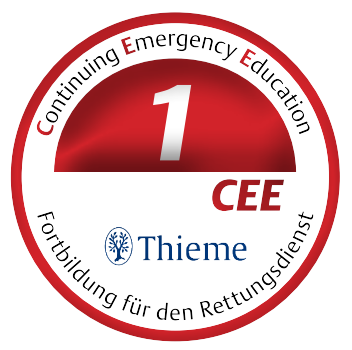

1

Wann ist eine Auskultation angezeigt?

bei der erweiterten Anamnese

falls in der Differenzialdiagnostik strittige Fragen auftreten

während des ABCDE-Schemas

am Ende des Ausfüllens vom Notfallprotokoll

wenn sich abzeichnet, dass ein Patient überhaupt transportiert wird

Welche Aussage zur Entstehung von Lungengeräuschen ist korrekt?

A Unter physiologischen Bedingungen ist die Strömung immer laminar und es sind fast keine Lungengeräusche auskultierbar.

B Die Atemgeräusche klingen in allen Bereichen der Lunge gleich.

C Über der Trachea hört man fast keine Strömungsgeräusche, da der Fluss hier nahezu vollständig laminar ist und es sich um eine große Röhre handelt, in der keine Turbulenzen entstehen.

D Atemgeräusche entstehen durch Turbulenzen der strömenden Luft und sind in der Phase des schnellsten Luftstroms einer Atemphase am besten wahrzunehmen.

E Verengungen der Bronchien - wie beim Asthma bronchiale - sind am besten am Ende der Einatmung zu hören, dies ist die sog. „silent lung“.

Welche Aussage zu pathologischen Atem-

\section{nebengeräuschen ist nicht korrekt?}

A Um Rasselgeräusche sicher als pathologisch beurteilen zu können, kann es Sinn machen, den Patienten vor der Auskultation husten zu lassen.

B Das Asthma cardiale zeichnet sich durch ein Pfeifen aus, das durch eine Kontraktion der Bronchialmuskulatur hervorgerufen wird.

C Brummen ist ein tief klingendes Atemnebengeräusch, das auf lange Schleimfäden hinweist.

D Das feuchte Rasselgeräusch eines Lungenödems ist in der Regel beidseits zu hören.

E Das feuchte Rasselgeräusch einer Lungenentzündung ist in der Regel zunächst an einer umschriebenen Stelle zu hören.

\section{Welche Aussage zu Herztönen ist korrekt?}

Eine Herzinsuffizienz beeinflusst die Lautstärke von Herztönen nicht.

B Bei der Systole ist die Mitralklappe geöffnet, bei der Diastole ist die Aortenklappe geöffnet.

C Bei höherer Herzfrequenz sind Geräusche leichter den beiden Herzphasen zuzuordnen.

D Mitralklappenvitien projizieren meist zur A. carotis.

E Bei der Systole ist die Aortenklappe geöffnet, bei der Diastole ist die Mitralklappe geöffnet.

Zu welchem Auskultationsbefund wird eine Pneumonie nicht führen?

seitenbetont

feuchte Rasselgeräusche

lokal begrenzt

inspiratorischer Stridor

Fieber
Welche Zuordnung auskultatorischer Befunde und anamnestischer Angaben stimmt nicht?

raues Systolikum im 2. ICR rechts und wiederkehrende Synkopen laut auskultierbares Giemen und plötzliches Auftreten nach Bienenstich aufgehobenes Atemgeräusch einseitig und plötzliche Schmerzen nach Husten

D beidseits feuchte Rasselgeräusche und plötzliches Auftreten aus der Ruhe

E normales Atemgeräusch bei subjektiver Atemnot, atemabhängigem Thoraxschmerz und Entlassung aus dem Krankenhaus nach einer Knieoperation am Vortag

\section{Welche Aussage ist korrekt?}

A Das häufigste Vitium ist die Aortenstenose, die häufigste Ursache sind entzündliche Erkrankungen.

B Das häufigste Vitium ist die Mitralinsuffizienz, die häufigste Ursache sind degenerative Prozesse.

C Das häufigste Vitium ist die Mitralinsuffizienz, die häufigste Ursache ist der Papillarmuskelabriss

D Das häufigste Vitium ist die Aortenstenose, die häufigste Ursache sind degenerative Prozesse.

E Das häufigste Vitium ist die Mitralstenose, die häufigste Ursache ist der i.v. Drogen-Abusus.

8 Welches der folgenden Geräuschphänomene gehört zur Trias nach Beck bei Herzbeuteltamponade?

rumpelnder erster Herzton

reibende Pleura

abgeschwächte Herztöne

fauchendes Systolikum

feinblasige ubiquitäre Rasselgeräusche

Auf welche Diagnostik bei V.a. Lungenentzündung kann man verzichten?

Messung der peripheren Sättigung

Messung der Körpertemperatur

Auszählung der Atemfrequenz

Messung von Herzfrequenz und Blutdruck

Kapnografie des spontan atmenden Patienten

Welche der folgenden Erkrankungen geht typischerweise nicht mit exspiratorischen Geräusche einher?

Asthma bronchiale

chronisch obstruktive Lungenerkrankung (COPD)

Spontanpneumothorax

akute Bronchitis

anaphylaktische Atemwegsschwellung

\section{cee.thieme.de}

- Sammeln Sie CEE-Punkte unter cee.thieme.de für Ihre Rettungsdienstfortbildung. 1 CEE-Punkt entspricht einer Fortbildungsstunde von 60 Minuten. 